\title{
Preparation of Highly Syndiotactic Poly(vinyl alcohol) by Fractionation
}

\author{
Tohei Yamamoto, ${ }^{\dagger}$ Koichi Kawakami, Takeshi Nishikawa, Mitsuhiro Sugimoto, \\ Nobuhiro KaWatsuki, Osamu Sangen, and Mikiharu KamaChi* \\ Faculty of Engineering, Himeji Institute of Technology, Shosha, Himeji 671-2201, Japan \\ *Faculty of Science, Osaka University, Machikaneyama, Toyonaka 560-0043, Japan
}

(Received September 26, 1997)

KEY WORDS Low Molecular Weight / Poly(vinyl alcohol) / Fractionation / Highly Syndiotactic /
Poly(vinyl pivalate) /

The physical properties of poly(vinyl alcohol) (PVA) are influenced by tacticity. We obtained highly syndiotactic PVA which showed higher crystal melting temperature and modulus than commercial PVA through radical polymerization of vinyl pivalate(VP). ${ }^{1}$ Hence we are interested in the synthesis of higher syndiotactic PVA.

Higher syndiotactic poly(vinyl ester)s, precursors of PVA, are obtained by a monomer with bulky substituent, by lowering polymerization temperature and using suitable solvent. Some studies on the solvent effects on the tacticities of poly(vinyl ester)s have been published. ${ }^{2-5}$ We studied the solvent effects on the tacticity of poly(vinyl pivalate) (PVP) and found that non polar solvents as $n$-alkane afforded higher syndiotactic PVA than polar solvents such as dimethyl sulfoxide. ${ }^{6}$

We consider that fractionation of PVA of low molecular weight is a good method to obtain highly syndiotactic PVA. Because we found through Monte Carlo simulation of sequence distribution that long racemo sequences appear frequently like block polymers and in the case of low molecular weight PVA, PVA molecules thus have very different tactic content, ${ }^{7}$ and consider that low molecular weight PVA of different tactic content shows different solubility and can be separated by fractional precipitation or fractional dissolution. In the case of high molecular weight PVA, as there is little tacticity difference among PVA molecules, and thus it is not possible to fractionate PVA molecules based on solubility differences.

Hence we first prepared low molecular weight PVA. Oxidative scission of 1,2 -glycol bond ${ }^{8}$ containing $1-2 \%$ in PVA and chain transfer reactions are considered usable for the preparation of low molecular weight PVA.

\section{EXPERIMENTAL}

\section{Preparation of Low Molecular Weight PVA}

The oxidative scission of commercially obtained PVA was carried out as follows. In $100 \mathrm{ml}$ of water, $5 \mathrm{~g}$ commercial PVA was dissolved at $70^{\circ} \mathrm{C}$ and $1.14 \mathrm{mmol}$ of $\mathrm{NaIO}_{4}$ was added to the solution and stirred for $30 \mathrm{~min}$. The PVA was precipitated into methanol, filtrated and dried under vacuum.

Radical polymerization of VP in isopropyl alcohol (IPA) having large chain transfer constants was car-

\footnotetext{
† To whom all correspondence should be addressed.
}

ried out as follows. VP, $\alpha, \alpha^{\prime}$-azobis(isobutyronitrile) (AIBN) and IPA purified by the usual method were placed in a Pyrex tube and the mixture was degassed to repeat the freezing-pumping-thawing cycle and sealed under vacuum. The tube was fixed in a thermostat bath at $-20^{\circ} \mathrm{C}$ and irradiated with a high pressure mercury lamp for a week. The polymer was precipitated in acetone, reprecipitated from acetone/water, filtrated and dried under vacuum. Saponification of PVP was carried out as in the previous paper. ${ }^{9}$

\section{Measurement}

The degree of polymerization $\left(P_{n}\right)$ was determined from the intrinsic viscosity of poly(vinyl acetate) (PVAc) derived from the PVA in acetone at $25^{\circ} \mathrm{C}$.

Triad tacticity was determined from the peak intensity of hydroxyl protons in the ${ }^{1} \mathrm{H}$ NMR spectrum of PVA measured with JEOL JNM EX-270.

Determination of the crystal melting temperature $\left(T_{\mathrm{m}}\right)$ was carried out using a Seiko SSC5200 scanning calorimeter.

\section{RESULTS AND DISCUSSION}

A PVA obtained by oxidative scission was fractionated to give fraction of $1 \%$ higher syndiotacticity. The terminal aldehyde groups reacted in the reacetylation reaction and determination of the PVA molecular weight was difficult. Hence we tried to make low molecular weight PVA using chain transfer reaction.

PVP obtained in IPA was saponified as before ${ }^{9}$ and afforded PVA of $P_{n}=c a .30$ determined from intrinsic viscosity of PVAc derived from PVA. Diad syndiotacticity was $67.8 \%$.

Table I. Characteristics of PVA fractionated from that derived from PVP obtained by radical polymerization in IPA

\begin{tabular}{|c|c|c|c|c|c|}
\hline \multirow{2}{*}{ PVA } & Fraction & \multirow{2}{*}{$P_{n}$} & \multirow{2}{*}{$\begin{array}{c}\text { Diad } \\
\text { syndio } \\
\text { tacticity } / \%\end{array}$} & \multirow{2}{*}{$\frac{T_{\mathrm{m}}^{\mathrm{a}}}{{ }^{\circ} \mathrm{C}}$} & \multirow{2}{*}{$\frac{\Delta H^{\mathrm{a}}}{\mathrm{mJ} \mathrm{mg^{-1 }}}$} \\
\hline & $\%$ & & & & \\
\hline Original & & 30 & 67.8 & 221 & 135 \\
\hline PVA-A & 9.0 & 80 & 69.6 & 238 & 150 \\
\hline PVA-B & 40.9 & 40 & 67.7 & 218 & 135 \\
\hline PVA-C & 34.3 & 30 & 67.2 & 211 & 131 \\
\hline
\end{tabular}

${ }^{a}$ Determined from DSC. 
PVA of $4.5 \mathrm{~g}$ was stirred with $500 \mathrm{ml}$ water under reflux and separated to insoluble part (PVA-A) and soluble part. The soluble part was separated in a insoluble part in cool water (PVA-B) and a soluble part in cool water (PVA-C). Triad tacticity was determined from the peak intensity of hydroxyl protons in the ${ }^{1} \mathrm{H}$ NMR spectrum of PVA and diad tacticity was derived from triad tacticity and listed in Table I.

As shown in Table I, the syndiotacticity of PVA-A was $c a .2 \%$ larger than the original, and $69.6 \%$ was the largest value obtained by radical polymerization. Although PVA-A has low $P_{n}$, it showed higher $T_{\mathrm{m}}$ and $\Delta H$ than the original. We consider the larger $P_{n}$ of PVA-A indicates lower solubility of PVA with larger $P_{n}$ but lower syndiotacticity. Not $100 \%$ yield in this study is considered as recovery loss of PVA with lower molecular weight and lower syndiotacticity.

We obtained PVA of higher syndiotacticity by fractionation. This supports the sequence distribution estimated by Monte Carlo method and indicates the possibility to obtain PVA of higher syndiotacticity by fractionation of low molecular weight PVA with high syndiotacticity. We believe it is possible to get PVA of much higher syndiotacticity by repeating fractionation.

\section{REFERENCES}

1. R. Fukae, T. Yamamoto, O. Sangen, T. Saso, and M. Kamachi, Polym. J., 22, 636 (1990).

2. U. Kador and P. Mehnert, Makromol. Chem., 144, 29 (1971).

3. S. Nozakura, M. Sumi, M. Uoi, T. Okamoto, and S. Murahashi, J. Polym. Sci., Polym. Chem. Ed., 11, 279 (1973).

4. K. Imai, T. Shiomi, N. Oda, and H. Otsuka, J. Polym. Sci., Polym. Chem. Ed., 24, 3225 (1986).

5. M. Matsuzawa, K. Yamaura, N. Noguchi, and H. Hayashi, Makromol. Chem., 165, 217 (1973).

6. R. Fukae, K. Kawakami, T. Yamamoto, O. Sangen, and M. Kamachi, Polym. J., 27, 1257 (1995); R. Fukae, T. Yamamoto, Y. Fujita, N. Kawatsuki, O. Sangen, and M. Kamachi, ibid., 29, 293 (1997).

7. T. Yamamoto, T. Isono, T. Matsumoto, M. Sugimoto, O. Sangen, and M. Kamachi, Polym. J., 27, 193 (1995)

8. J. E. Mulvaney and R. G. Gollmar, J. Polym. Sci., A-1, 3667 (1970).

9. T. Yamamoto, S. Yoda, H. Takase, T. Saso, O. Sangen, R. Fukae, M. Kamachi, and T. Sato, Polym. J., 23, 185 (1991). 